\title{
Erratum to: Elemental diet plus glutamine for the prevention of mucositis in esophageal cancer patients receiving chemotherapy: a feasibility study
}

\author{
Yoshihiro Tanaka $^{1}$ - Takao Takahashi ${ }^{1} \cdot$ Kazuya Yamaguchi $^{1} \cdot$ Shinji Osada $^{1}$. $^{-}$ \\ Toshio Shimokawa ${ }^{2} \cdot$ Kazuhiro Yoshida $^{1}$
}

Published online: 5 November 2015

(C) Springer-Verlag Berlin Heidelberg 2015

Erratum to: Support Care Cancer

DOI 10.1007/s00520-015-2864-9

Table 2. The number of patients in Gln and Gln plus ED

should be corrected.

This is the correct Table 2.

Table 2 Multivariate analysis for independent factors affecting increased mucositis grade during chemotherapy

\begin{tabular}{|c|c|c|c|c|c|c|}
\hline Factor & & $n$ & Odds ratio $(95 \% \mathrm{CI})$ & $P$ value & Odds ratio $(95 \% \mathrm{CI})$ & $P$ value \\
\hline \multirow[t]{2}{*}{ Gln } & With & 10 & $4.1(0.6-33.7)$ & 0.17 & $4.0(0.7-29.6)$ & 0.15 \\
\hline & Without & 20 & & & & \\
\hline \multirow[t]{2}{*}{ Gln plus ED } & With & 10 & $0.1(0.0-0.7)$ & $0.03^{*}$ & $0.1(0.0-0.6)$ & $0.02 *$ \\
\hline & Without & 20 & & & & \\
\hline \multirow[t]{2}{*}{ Age, years } & $<70$ & 16 & $3.1(0.7-16.5)$ & 0.16 & $3.1(0.7-16.1)$ & 0.15 \\
\hline & $\geq 70$ & 14 & & & & \\
\hline \multirow[t]{2}{*}{ Sex } & Male & 26 & $0.9(0.1-7.7)$ & 0.95 & - & - \\
\hline & Female & 4 & & & & \\
\hline \multirow[t]{2}{*}{ Cancer stage } & IVa,b & 13 & $14.6(1.9-150.0)$ & $0.01^{*}$ & $13.3(1.9-123.2)$ & $0.01 *$ \\
\hline & II-III & 17 & & & & \\
\hline \multirow[t]{2}{*}{ Treatment } & First-line & 7 & $0.2(0.0-2.1)$ & 0.19 & $0.2(0.0-1.6)$ & 0.13 \\
\hline & NAC & 23 & & & & \\
\hline \multirow[t]{2}{*}{ BMI } & $<21$ & 13 & $1.9(0.4-9.6)$ & 0.44 & - & - \\
\hline & $\geq 21$ & 17 & & & & \\
\hline
\end{tabular}

$B M I$ body mass index, $E D$ elemental diet, Gln glutamine, $N A C$ neoadjuvant chemotherapy

${ }^{*} p<0.05$

The online version of the original article can be found at http://dx.doi.org/ 10.1007/s00520-015-2864-9.

Yoshihiro Tanaka

yoshihirotana11@hotmail.com

1 Department of Surgical Oncology, Gifu Graduate School of Medicine, 1-1 Yanagido, Gifu City, Gifu 501-1194, Japan

2 Graduate School of Medicine and Engineering, University of Yamanashi, 4-4-37 Takeda, Kofu City 400-8510, Japan 